\title{
Tuberculous tenosynovitis
}

\author{
Clayton J. Sanders, MD, and William G. Schucany, MD
}

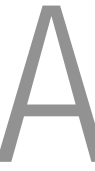

29-year-old African American woman presented with pain and swelling of the hand and wrist with a lowgrade fever. She was on immunosuppressive therapy for systemic lupus erythematosus. Plain radiographs (Figure 1) revealed inflammatory arthritis related to lupus, with subluxations at the metacarpophalangeal joints and radial deviation of the fingers. Classically, the joint space was preserved, osseous erosions were absent, and bone density was near normal.

Magnetic resonance (MR) images (Figures 2-8) showed a large effusion of the flexor tendon sheaths of both the hand

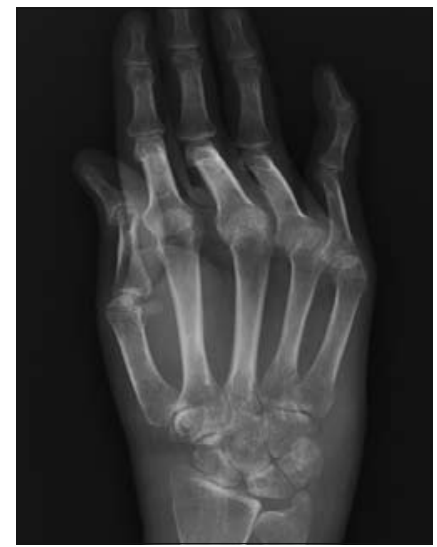

Figure 1. Plain radiograph of the hand reveals subluxations at the metacarpophalangeal joints with boutonniere deformities of the digits. No erosions or joint space narrowing are evident.

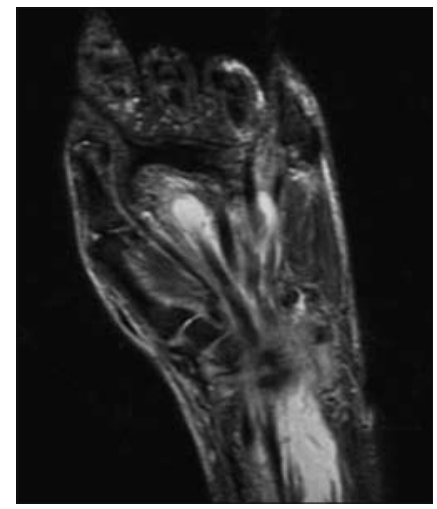

Figure 3. Coronal short-inversion-time inversion recovery (STIR) image demonstrates a large tendon sheath effusion tracking along the flexor tendons of the hand and wrist.

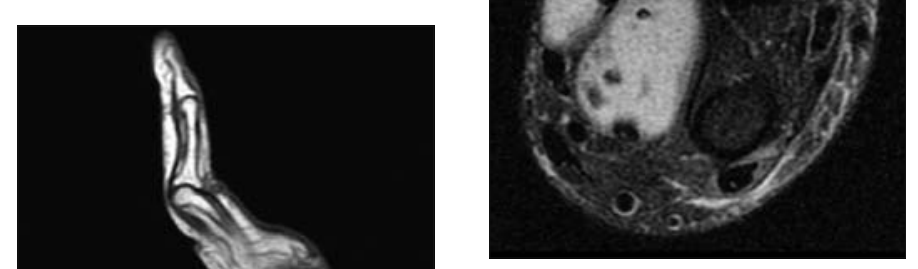

Figure 5. Axial STIR image through the wrist shows a large flexor tendon sheath effusion with inflammatory debris and rice bodies layering in the dependent portion of the effusion.

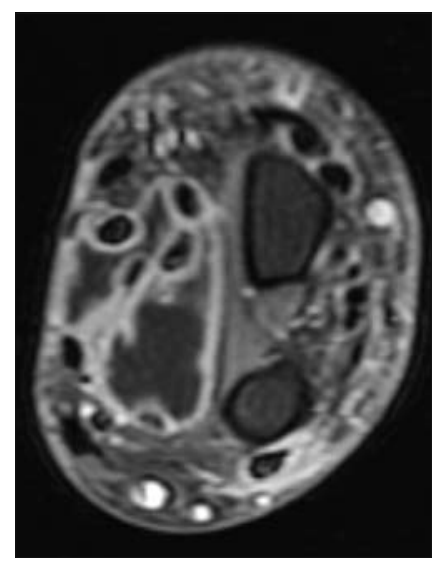

Figure 7. Axial fat-saturated T1weighted image with gadolinium contrast at the level of the wrist demonstrates a large effusion of the flexor tendon sheath with thick, smoothly marginated peripheral enhancement.

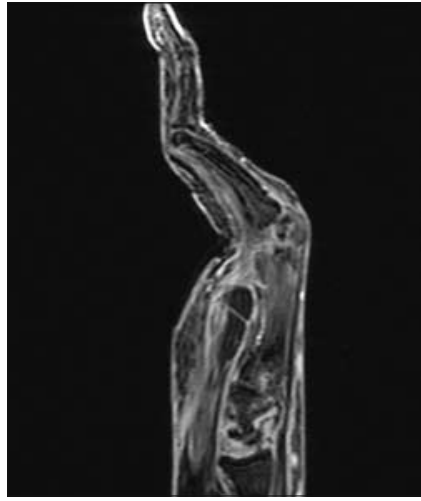

Figure 6. Sagittal fat-saturated T1weighted image with contrast reveals a smoothly marginated peripherally enhancing effusion of the flexor tendon sheath of the hand.

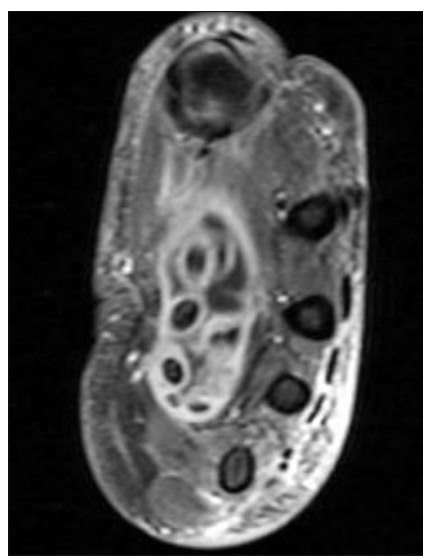

Figure 8. Axial fat-saturated T1weighted image with contrast at the level of the mid-hand again demonstrates a large effusion of the flexor tendon sheath with peripheral enhancement.
Figure 4. Axial STIR image through the hand reveals a large flexor tendon sheath effusion.
From the Department of Radiology, Baylor University Medical Center, Dallas, Texas. Corresponding author: William G. Schucany, MD, Department of Radiology, Baylor University Medical Center, 3500 Gaston Avenue, Dallas, Texas 75246 (e-mail: gschucany@americanrad.com). 
DIAGNOSIS: The culture of the synovial fluid grew Mycobacterium tuberculosis, and the patient was placed on antibiotics.

\section{DISCUSSION}

The incidence of tuberculosis has been increasing steadily since the early 1990s, predominantly in poverty-stricken inner-city areas and in the immunocompromised. In 2004, the incidence of tuberculosis in the USA was approximately 4.9 active cases in 100,000. Mycobacterium tuberculosis remains a top-10 cause of death worldwide, with greater than 2 billion active cases occurring mostly in developing countries.

Tuberculosis is spread primarily by inhalation of infected droplets, resulting in pulmonary disease, although rare cases of gastrointestinal and transdermal spread have been reported. Approximately $1 \%$ to $3 \%$ of patients infected with tuberculosis demonstrate skeletal involvement, most often manifested as spondylitis, peripheral arthritis, or osteomyelitis. Approximately $50 \%$ of patients with musculoskeletal tuberculosis have concomitant pulmonary disease. Tuberculous tenosynovitis is a rare complication of the disease, typically resulting from direct extension from adjacent bone or joint infection, although hematogenous spread from a distant primary focus is also possible.

A well-known complication of tuberculous arthritis and tenosynovitis is the production of a "cold" abscess. The abscess is termed "cold" due to the lack of production of proteolytic enzymes, which results in cartilage destruction. Characteristic features of tuberculous arthritis include osseous erosions in the absence of subchondral marrow signal abnormality. The erosions result from profuse pannus formation and granulation tissue. Extra-articular extension of the infection results in a soft tissue mass that shows smooth margins with thin, homogeneous enhancement.

The onset of tuberculous tenosynovitis is gradual, with progressive swelling, pain, and diminished range of motion. Tendon sheaths of the hand and wrist are most frequently involved. The disease takes on three stages as it progresses: the earliest hygromatous form, a serofibrinous form, and a fungoid form, with considerable overlap of the three stages at presentation. The hygromatous form usually appears as a serous exudate within a normal-appearing tendon sheath; however, the tendon sheath may be thinned or replaced by granulation tissue. As the infection evolves, the serofibrinous form is manifested by obliteration of the tendon sheath with fibrous tissue and caseous inflammatory debris. Rice bodies appear in the synovial fluid, and involvement of the tendon itself with granulation tissue is seen. Intertendinous adhesions may form, or complete rupture may occur. The fungoid stage involves extensive caseation and granulation tissue formation, causing obliteration of the tendon and sheath with formation of sinus tracts and a cold abscess. Cold abscesses are frequently associated with bone and joint involvement.

Plain radiographs generally demonstrate soft tissue swelling with or without calcification. Osteopenia may be observed, indicating areas of hyperemia. In chronic cases, joint space narrowing and osseous erosions may be seen.

MR imaging allows assessment of the entire tendon and sheath. During the hygromatous form of the disease, a serous exudate indicating a nonspecific tenosynovitis is likely to be the only finding. The serofibrinous stage results in thickened synovium with low signal intensity material within the synovial fluid on T2 images. Tendon involvement manifested by tendon thinning, tethering, or disruption may be seen during this stage. Extension beyond the tendon sheath with enhancing soft tissue mass formation is characteristic of the fungoid stage.

The differential diagnosis for tuberculous tenosynovitis includes rheumatoid arthritis, pyogenic infection, gouty arthritis, giant cell tumor of the tendon sheath, and fungal tenosynovitis. Diagnosis of articular involvement depends on the isolation of the organism from synovial fluid or synovial biopsy. Prompt diagnosis is critical to preserve joint function and anatomy.

\section{General references}

1. Hong SH, Kim SM, Ahn JM, Chung HW, Shin MJ, Kang HS. Tuberculous versus pyogenic arthritis: MR imaging evaluation. Radiology 2001;218(3):848-853.

2. Jaovisidha S, Chen C, Ryu KN, Siriwongpairat P, Pekanan P, Sartoris DJ, Resnick D. Tuberculous tenosynovitis and bursitis: imaging findings in 21 cases. Radiology 1996;201(2):507-513.

3. Yao DC, Sartoris DJ. Musculoskeletal tuberculosis. Radiol Clin North Am 1995;33(4):679-689.

4. Sommer OJ, Kladosek A, Weiler V, Czembirek H, Boeck M, Stiskal M. Rheumatoid arthritis: a practical guide to state-of-the-art imaging, image interpretation, and clinical implications. Radiographics 2005;25(2): 381-398.

5. World Health Organization. Estimated TB incidence, prevalence and mortality, 2005. Last updated March 2007. Available at http://www.who. int/mediacentre/factsheets/fs104/en/; accessed October 24, 2007. 